This is the peer reviewed version of the following article:

Radonjic Aleksandar, "Integer codes correcting single asymmetric errors" Annals of

Telecommunications, 76, no. 1-2 (2021):109-113, https://doi.org/10.1007/s12243-020-00816-w

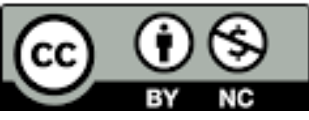

I. This work is licensed under a

Attribution-NonCommercial 4.0 International (CC BY-NC 4.0) 


\title{
Integer Codes Correcting Single Asymmetric Errors
}

\author{
Aleksandar Radonjic \\ Institute of Technical Sciences of the Serbian Academy of Sciences and Arts, Belgrade, Serbia \\ E-mail: sasa_radonjic@yahoo.com
}

Abstract: This paper presents a class of integer codes capable of correcting single asymmetric errors. The presented codes are defined over the ring of integers modulo $2^{b}-1$ and are constructed with the help of a computer. The results of an exhaustive search have shown that, for practical lengths up to 4096 bits, the proposed codes use the same number of check-bits as the best systematic single asymmetric error correcting codes (SAECCs). Besides this, it is found that for some lengths the presented codes are perfect. Finally, the paper shows that the encoding/decoding complexity of the proposed codes is notably lower than that of the best systematic SAECCs.

Keywords: Integer codes, single asymmetric errors, error correction, perfect codes.

\section{Introduction}

Conventional coding theory is mainly focused on constructing codes for use over channels in which $1 \rightarrow 0$ and $0 \rightarrow 1$ errors occur with equal probability. However, it is known that some channels display only $1 \rightarrow 0$ errors. For example, in optical communications, photons may fade or fail to be detected ( $1 \rightarrow 0$ errors), but the creation of spurious photons $(0 \rightarrow 1$ errors) is not possible [1]. Likewise, in some VLSI circuits and memories charges may leak with time $(1 \rightarrow 0$ errors), but new charges cannot be spontaneously created $(0 \rightarrow 1$ errors $)$ [2].

Motivated by these and similar examples, researchers began constructing codes that correct asymmetric $(1 \rightarrow 0)$ errors. Among them, special attention has been paid to the construction of single asymmetric error correcting codes (SAECCs). The reason for this was an attempt to design codes that would have higher rates than Hamming codes [3]. Although some successes have been achieved in the case of non-systematic codes [4]-[12], to date none systematic SAECC has been constructed that outperforms Hamming codes. This is not surprising given that Bose and Al-Bassam [13] showed that the best systematic SAECCs have the same parameters as Hamming codes, except possibly for the lengths $n=2^{u}$ and $n=2^{u}+1$, where $u \geq 4$. In these two cases, as they stated, there may exist systematic SAECCs that are better than Hamming codes. However, such codes were never reported in the literature. The only known systematic SAECCs are those designed by Abdel-Ghaffar and Ferreira [14]. These codes are obtained by modifying group-theoretic (GT) codes [2], which means that they have the same parameters as Hamming codes. 
In this paper, we will present a class of systematic SAECCs that are significantly different from the codes proposed in [14]. The main difference is that the presented codes are not binary oriented, but are defined over the ring of integers modulo $2^{b}-1$. In addition, unlike [14], they are constructed with the help of a computer. One consequence of these differences is that our codes are perfect only for certain lengths. However, as we will see, for all practical lengths up to 4096 bits, they use the same number of check-bits as the codes from [14].

The organization of this paper is as follows: Section 2 deals with the construction of integer codes capable of correcting single asymmetric errors. In Section 3, the proposed codes are evaluated and compared with the best systematic SAECCs, while Section 4 concludes the paper.

\section{Integer SAEC Codes}

\section{A. Codes Construction}

As stated previously, the only known systematic SAECCs are those obtained by modifying GT codes. According to [14], a modified GT (MGT) code can be defined as

$$
C(n, d)=\left\{u \in\{0,1\}^{n}: \sum_{i=1}^{K} d_{i} \cdot u_{i} \equiv d\right\}
$$

where $u=\left(u_{1}, \ldots, u_{K}, u_{K+1}, \ldots, u_{n}\right) \in\{0,1\}^{n}$ is the codeword vector, $d_{i}$ is the element of the Abelian group $G$ of the order $K+1$ and $d=\sum_{i=K+1}^{n} u_{i} \cdot 2^{i-K+1}$ is a fixed integer in $G$. Unlike MGT codes, the presented ones are defined over the ring of integers modulo $2^{b}-1$. This is formally described by the following definitions.

Definition 1. [15] Let $Z_{2^{b}-1}=\left\{0,1, \ldots, 2^{b}-2\right\}$ be the ring of integers modulo $2^{b}-1$ and let $B_{i}=\sum_{n=0}^{b-1} a_{n} \cdot 2^{n}$ be the integer representation of a $b$-bit byte, where $a_{n} \in\{0,1\}$ and $1 \leq i \leq k$. Then, the code $C(b, k, c)$, defined as

$$
C(b, k, c)=\left\{x \in Z_{2^{b}-1}^{k+1}: \sum_{i=1}^{k} C_{i} \cdot B_{i} \equiv B_{k+1}\left(\bmod 2^{b}-1\right)\right\}
$$

is an $(k b+b, k b)$ integer code, where $x=\left(B_{1}, B_{2}, \ldots, B_{k}, B_{k+1}\right) \in Z_{2^{b}-1}^{k+1}$ is the codeword vector, $c=$ $\left(C_{1}, C_{2}, \ldots, C_{k}, 1\right) \in Z_{2^{b}-1}^{k+1}$ is the coefficient vector and $B_{k+1} \in Z_{2^{b}-1}$ is an integer.

Definition 2. [16] Let $x=\left(B_{1}, B_{2}, \ldots, B_{k}, B_{k+1}\right) \in Z_{2^{b}-1}^{k+1}, y=\left(\underline{B}_{1}, \underline{B}_{2}, \ldots, \underline{B}_{k}, \underline{B}_{k+1}\right) \in Z_{2^{b}-1}^{k+1}$ and $e=\left(\underline{B}_{1}-B_{1}, \underline{B}_{2}-B_{2}, \ldots, \underline{B}_{k}-B_{k}, B_{k+1}-\underline{B}_{k+1}\right)=\left(e_{1}, e_{2}, \ldots, e_{k}, e_{k+1}\right) \in Z_{2^{b}-1}^{k+1}$ be respectively, the sent codeword, the received codeword and the error vector. Then, the syndrome $S$ of the received codeword is defined as

$$
S=\sum_{i=1}^{k} C_{i} \cdot \underline{B}_{i}-\underline{B}_{k+1}\left(\bmod 2^{b}-1\right)=\sum_{i=1}^{k+1} e_{i} \cdot C_{i}\left(\bmod 2^{b}-1\right)
$$

Definition 3. An $(k b+b, k b)$ integer code is called $S A E C$ if it can correct error vectors from the set $\varepsilon=\left\{\left(-2^{r}, 0, \ldots, 0,0\right), \ldots,\left(0,0, \ldots,-2^{r}, 0\right),\left(0,0, \ldots, 0,2^{r}\right)\right\}$, where $1 \leq r \leq b-1$. 
Definition 4. The error set for $(k b+b, k b)$ integer SAECCs is defined by

$\xi_{b, k}=s_{1} \mathrm{U} s_{2}$

where

$$
\begin{aligned}
& s_{1}=\left\{-2^{r} \cdot C_{i}\left(\bmod 2^{b}-1\right): 0 \leq r \leq b-1,1 \leq i \leq k\right\} \\
& s_{2}=\left\{2^{r}: 0 \leq r \leq b-1\right\}
\end{aligned}
$$

From the above it is obvious that integer SAECCs cannot be constructed without knowing the values of the $C_{i}$ 's. This fact, however, does not prevent us to state the following theorems.

Theorem 1. An $(k b+b, k b)$ integer SAECC exists only if $\left|\xi_{b, k}\right|=b \cdot(k+1)$, where $\left|\xi_{b, k}\right|$ denotes the cardinality of $\xi_{b, k}$.

Proof. Observe that the set $\xi_{b, k}$ can be expressed as

$$
\xi_{b, k}=\bigcup_{i=1}^{k+1} m_{i}
$$

where

$$
\begin{aligned}
& m_{1}=\left\{-2^{r} \cdot C_{1}\left(\bmod 2^{b}-1\right): 0 \leq r \leq b-1\right\}, \\
& \text { M } \\
& m_{k}=\left\{-2^{r} \cdot C_{k}\left(\bmod 2^{b}-1\right): 0 \leq r \leq b-1\right\} \text {, } \\
& m_{k+1}=\left\{2^{r}: 0 \leq r \leq b-1\right\} \text {. }
\end{aligned}
$$

The elements of the above subsets will be nonzero and mutually different only if the coefficients $C_{i}$ have values such that

$m_{1}$ I L I $m_{k}$ I $m_{k+1}=\varnothing$, $\left|m_{1}\right|=\mathrm{L}=\left|m_{k}\right|=\left|m_{k+1}\right|$.

As a result, it follows that $\left|\xi_{b, k}\right|=\left|m_{1}\right|+\mathrm{L}+\left|m_{k}\right|+\left|m_{k+1}\right|=\left|m_{k+1}\right| \cdot(k+1)=b \cdot(k+1)$.

Theorem 2. For any $(k b+b, k b)$ integer SAECC it holds that $k \leq\left\lfloor\frac{2^{b}-b-2}{b}\right\rfloor$.

Proof. From Theorem 1 we know that the set $\xi_{b, k}$ has $b \cdot(k+1)$ nonzero elements. On the other hand, Definition 1 says that the total number of nonzero syndromes is equal to $2^{b}-2$. Obviously, we have the inequality

$b \cdot(k+1) \leq 2^{b}-2$

wherefrom it follows that $k \leq\left\lfloor\frac{2^{b}-b-2}{b}\right\rfloor$. 
Theorem 3. Any perfect $(k b+b, k b)$ integer $S A E C C$, if exists, has a rate of $\left(2^{b}-b-2\right) /\left(2^{b}-2\right)$.

Proof. This theorem follows directly from Theorem 2.

Table 1. Number of Coefficients for Integer SAECCs with Parameter $b \leq 12$.

\begin{tabular}{||c|c|c|c|c|c|c|c|c|c|c||}
\hline & $b=3$ & $b=4$ & $b=5$ & $b=6$ & $b=7$ & $b=8$ & $b=9$ & $b=10$ & $b=11$ & $b=12$ \\
\hline Bound & 1 & 2 & 5 & 9 & 17 & 30 & 55 & 101 & 185 & 340 \\
\hline Experiment & 1 & 2 & 5 & 8 & 17 & 29 & 55 & 98 & 185 & 334 \\
\hline
\end{tabular}

Table 2. Coefficients for Integer SAECCs with Parameter $b \leq 12$.

\begin{tabular}{|c|c|c|c|c|c|c|c|c|c|c|c|c|c|c|c|c|c|c|c|c|}
\hline \multicolumn{21}{|c|}{$b=3$} \\
\hline 2 & & & & & & & & & & & & & & & & & & & & \\
\hline \multicolumn{21}{|c|}{$b=4$} \\
\hline 2 & 3 & & & & & & & & & & & & & & & & & & & \\
\hline \multicolumn{21}{|c|}{$b=5$} \\
\hline 2 & 3 & 5 & 7 & 11 & & & & & & & & & & & & & & & & \\
\hline \multicolumn{21}{|c|}{$b=6$} \\
\hline 2 & 3 & 5 & 7 & 11 & 1 & & 15 & 23 & & & & & & & & & & & & \\
\hline \multicolumn{21}{|c|}{$b=7$} \\
\hline 2 & 3 & 5 & 7 & 9 & 1 & & 13 & 15 & 19 & 21 & 23 & 27 & 29 & 31 & 43 & 47 & 55 & & & \\
\hline \multicolumn{21}{|c|}{$b=8$} \\
\hline 2 & 3 & 5 & 7 & 9 & 1 & & 13 & 15 & 19 & \begin{tabular}{l|}
21 \\
\end{tabular} & 23 & 25 & 27 & 29 & 31 & 37 & 39 & 43 & 45 & 47 \\
\hline 53 & 55 & 59 & 61 & 63 & 8 & & 91 & 95 & 111 & & & & & & & & & & & \\
\hline \multicolumn{21}{|c|}{$b=9$} \\
\hline 2 & 3 & 5 & 7 & 9 & 1 & & 13 & 15 & 17 & \begin{tabular}{l|l}
19 \\
\end{tabular} & 21 & 23 & 25 & 27 & 29 & 31 & 35 & 37 & 39 & 41 \\
\hline 43 & 45 & 47 & 51 & 53 & 5 & & 57 & 59 & 61 & 63 & 75 & 77 & 79 & 83 & 85 & 87 & 91 & 93 & 95 & 103 \\
\hline 107 & 109 & 111 & 117 & 119 & 12 & & 125 & 127 & 171 & 175 & 183 & 187 & 191 & 223 & 239 & & & & & \\
\hline \multicolumn{21}{|c|}{$b=10$} \\
\hline 2 & 3 & 5 & 7 & 9 & 1 & & 13 & 15 & 17 & 19 & 21 & 23 & 25 & 27 & 29 & 31 & 35 & 37 & 39 & 41 \\
\hline 43 & 45 & 47 & 49 & 51 & 5 & & 55 & 57 & 59 & 61 & 63 & 69 & 71 & 73 & 75 & 77 & 79 & 83 & 85 & 87 \\
\hline 89 & 91 & 93 & 95 & 101 & 10 & & 105 & 107 & 109 & 111 & 115 & 117 & 119 & 121 & 123 & 125 & 127 & 147 & 149 & 151 \\
\hline 155 & 157 & 159 & 167 & 171 & 17 & & 175 & 179 & 181 & 183 & 187 & 189 & 191 & 205 & 207 & 213 & 215 & 219 & 221 & 223 \\
\hline 235 & 237 & 239 & 245 & 247 & 25 & & 253 & 255 & 343 & 347 & 351 & 367 & 375 & 379 & 383 & 439 & 447 & 479 & & \\
\hline \multicolumn{21}{|c|}{$b=11$} \\
\hline 2 & 3 & 5 & 7 & 9 & 1 & & 13 & 15 & 17 & \begin{tabular}{l|l}
19 \\
\end{tabular} & 21 & 23 & 25 & 27 & 29 & 31 & 33 & 35 & 37 & 39 \\
\hline 41 & 43 & 45 & 47 & 49 & 5 & & 53 & 55 & 57 & 59 & 61 & 63 & 67 & 69 & 71 & 73 & 75 & 77 & 79 & 81 \\
\hline 83 & 85 & 87 & 89 & 91 & 9 & & 95 & 99 & 101 & 103 & 105 & 107 & 109 & 111 & 113 & 115 & 117 & 119 & 121 & 123 \\
\hline 125 & 127 & 137 & 139 & 141 & 14 & & 147 & 149 & 151 & 153 & 155 & 157 & 159 & 163 & 165 & 167 & 169 & 171 & 173 & 175 \\
\hline 179 & 181 & 183 & 185 & 187 & 18 & & 191 & 199 & $\frac{1}{201}$ & 203 & 205 & 207 & 211 & 213 & 215 & 217 & 219 & 221 & 223 & 229 \\
\hline 231 & 233 & 235 & 237 & 239 & 24 & & 245 & 247 & 249 & 251 & 253 & 255 & 293 & 295 & 299 & 301 & 303 & 307 & 309 & 311 \\
\hline 315 & 317 & 319 & 331 & 333 & 33 & & 339 & 341 & 343 & 347 & 349 & 351 & 359 & 363 & 365 & 367 & 371 & 373 & 375 & 379 \\
\hline 381 & 383 & 411 & 413 & 415 & 42 & & 427 & 429 & 431 & 437 & 439 & 443 & 445 & 447 & 463 & 469 & 471 & 475 & 477 & 479 \\
\hline 491 & 493 & 495 & 501 & 503 & 50 & & 509 & 511 & 683 & 687 & 695 & 699 & 703 & 727 & 731 & 735 & 751 & 759 & 763 & 767 \\
\hline 879 & 887 & 895 & 959 & 991 & & & & & & & & & & & & & & & & \\
\hline \multicolumn{21}{|c|}{$b=12$} \\
\hline 2 & 3 & 5 & 7 & 9 & 1 & & 13 & 15 & 17 & \begin{tabular}{l|l}
19 \\
\end{tabular} & 21 & 23 & 25 & 27 & 29 & 31 & 33 & 35 & 37 & 39 \\
\hline 41 & 43 & 45 & 47 & 49 & 5 & & 53 & 55 & 57 & 59 & 61 & 63 & 67 & 69 & 71 & 73 & 75 & 77 & 79 & 81 \\
\hline 83 & 85 & 87 & 89 & 91 & 9 & & 95 & 97 & 99 & 101 & 103 & 105 & 107 & 109 & 111 & 113 & 115 & 117 & 119 & 121 \\
\hline 123 & 125 & 127 & 133 & 135 & 13 & & 139 & 141 & 143 & 145 & 147 & 149 & 151 & 153 & 155 & 157 & 159 & 163 & 165 & 167 \\
\hline 169 & 171 & 173 & 175 & 177 & 17 & & 181 & 183 & 185 & 187 & 189 & 191 & 197 & 199 & 201 & 203 & 205 & 207 & 209 & 211 \\
\hline 213 & 215 & 217 & 219 & 221 & 22 & & 227 & 229 & 231 & 233 & 235 & 237 & 239 & 241 & 243 & 245 & 247 & 249 & 251 & 253 \\
\hline 255 & 275 & 277 & 279 & 281 & 28 & & 285 & 287 & 291 & 293 & 295 & 297 & 299 & 301 & 303 & 307 & 309 & 311 & 313 & 315 \\
\hline 317 & 319 & 327 & 329 & 331 & 33 & & 335 & 339 & 341 & 343 & 345 & 347 & 349 & 351 & 355 & 357 & 359 & 361 & 363 & 365 \\
\hline 367 & 371 & 373 & 375 & 377 & 37 & & 381 & 383 & 397 & 399 & 403 & 405 & 407 & 409 & 411 & 413 & 415 & 421 & 423 & 425 \\
\hline 427 & 429 & 431 & 435 & 437 & 43 & & 441 & 443 & 445 & 447 & 457 & 459 & 461 & 463 & 467 & 469 & 471 & 473 & 475 & 477 \\
\hline 479 & 485 & 487 & 489 & 491 & 49 & & 495 & 499 & 501 & 503 & 505 & 507 & 509 & 511 & 587 & 589 & 591 & 595 & 597 & 599 \\
\hline 603 & 605 & 607 & 613 & 615 & 61 & & 621 & 623 & 627 & 629 & 631 & 635 & 637 & 639 & 661 & 663 & 667 & 669 & 671 & 679 \\
\hline 683 & 685 & 687 & 691 & 693 & 69 & & 699 & 701 & 703 & 717 & 719 & 723 & 725 & 727 & 731 & 733 & 735 & 743 & 747 & 749 \\
\hline 751 & 755 & 757 & 759 & 763 & 76 & & 767 & 821 & 823 & 827 & 829 & 831 & 847 & 853 & 855 & 859 & \begin{tabular}{|l|}
861 \\
\end{tabular} & 863 & 871 & 875 \\
\hline 877 & 879 & 885 & 887 & 891 & 89 & & 895 & 925 & 927 & 939 & 941 & 943 & 949 & 951 & 955 & 957 & \begin{tabular}{|l|}
959 \\
\end{tabular} & 981 & 983 & 987 \\
\hline 989 & 991 & 100 & 100 & 1007 & 10 & & 1015 & 1019 & 1021 & 1023 & 1367 & 1371 & 1375 & 1387 & 1391 & 1399 & 1403 & 1407 & 1455 & 1463 \\
\hline 1467 & $\mid 1471$ & 149 & 150 & 1519 & 15 & & 1531 & 1535 & 1759 & 1775 & 1783 & 1791 & 1919 & 1983 & & & & & & \\
\hline
\end{tabular}


The last step in constructing integer SAECCs is to find the $C_{i}$ 's that satisfy the condition of Theorem 1. For that purpose it is necessary to perform an exhaustive search on all possible candidates from the set $Z_{2^{b}-1} \backslash\{0,1\}$. In this paper, we have restricted ourselves to values of $b$ less than or equal to 12 . The reason for this is twofold: first, the number of the $C_{i}$ 's roughly doubles with the increase of $b$ (Tables 1 and 2), and second, for the mentioned values of $b$ the proposed codes are fully comparable with those presented in [15].

\section{B. Error Correction Procedure}

The error correction procedure for the presented codes is very similar to those described in [15], [16]. In short, if $S \neq 0$, the decoder will lookup the syndrome table (ST) to find the entry with the error correction data. After that, in the next step, it will execute the operation

$$
B_{i}=\underline{B}_{i}+\underline{E}\left(\bmod 2^{b}-1\right)
$$

where $\underline{E} \in\left\{2^{r}: 0 \leq r \leq b-1\right\}$. To generate the ST it is necessary to substitute the values of $b$ and $C_{i}$ into (5)-(6). In this way, exactly $\left|\xi_{b, k}\right|$ (Theorem 1) relationships (Fig. 1) between the nonzero syndrome (element of the set $\xi_{b, k}$ ), error location $(i)$ and error vector $(\underline{E})$ will be established. So, when $S \neq 0$, the decoder's task will be to find the entry with the first $b$ bits as that of the syndrome $S$.

\begin{tabular}{|l|l|l|}
\hline Element of the set $\xi_{b, k}(S)$ & Error location $(i)$ & Error vector $(\underline{\underline{E}})$ \\
\hline & & \\
\hline & $-\left\lceil\log _{2}(k+1)\right\rceil \rightarrow$ & \\
\hline
\end{tabular}

Fig. 1. Bit-width of one syndrome table entry.

Example 1. Let $b=5, k=5, C_{1}=2, C_{2}=3, C_{3}=5, C_{4}=7$ and $C_{5}=11$. According to Theorem 1, the ST will have $\left|\xi_{5,5}\right|=25$ entries (Table 3). Now, suppose that the encoder needs to encode 25 data bits, $\mathrm{D}=10101110011001000110$ 01010. From (3) we know that the value of the last (sixth) byte will be equal to $B_{k+1}=B_{6}=2 \cdot 21+3 \cdot 25+5 \cdot 18+7 \cdot 6+11 \cdot 10(\bmod 31)=18=10010_{2}$ the codeword will have the form $x=\left(B_{1}, B_{2}, B_{3}, B_{4}, B_{5}, B_{6}\right)=\left(10101_{2}, 11001_{2}, 10010_{2}, 00110_{2}\right.$, $\left.01010_{2}, 10010_{2}\right)=(21,25,18,6,10,18)$. Assume now that the 5 th bit is flipped. In that case, the codeword will have the form $y=\left(\underline{B}_{1}, \underline{B}_{2}, \underline{B}_{3}, \underline{B}_{4}, \underline{B}_{5}, \underline{B}_{6}\right)=\left(1010 \underline{0}_{2}, 11001_{2}, 10010_{2}, 00110_{2}\right.$, $\left.01010_{2}, 10010_{2}\right)=(20,25,18,6,10,18)$. As explained previously, the decoder will perform the operation

$S=2 \cdot 20+3 \cdot 25+5 \cdot 18+7 \cdot 6+11 \cdot 10-18(\bmod 31)=29$

after which it will conclude that the first byte is in error (Table 3). As a result, it will execute the operation

$B_{1}=20+1(\bmod 31)=21$. 
Table 3. The ST for the Perfect $(30,25)$ Integer SAEC Code.

\begin{tabular}{|c|c|c|c|c|c|c|c|c|c|c|c|c|c|c|c|c|c|c|c|}
\hline & $S$ & $i$ & $\underline{E}$ & & $S$ & $i$ & $\underline{E}$ & & $S$ & $i$ & $\underline{E}$ & & $S$ & $i$ & $\underline{E}$ & & $S$ & $i$ & $\underline{E}$ \\
\hline 1 & 1 & 6 & 1 & 7 & 7 & 2 & 8 & 13 & 13 & 3 & 16 & 19 & 19 & 2 & 4 & 25 & 25 & 2 & 2 \\
\hline 2 & 2 & 6 & 2 & 8 & 8 & 6 & 8 & 14 & 14 & 2 & 16 & 20 & 20 & 5 & 1 & 26 & 26 & 3 & 1 \\
\hline 3 & 3 & 4 & 4 & 9 & 9 & 5 & 2 & 15 & 15 & 1 & 8 & 21 & 21 & 3 & 2 & 27 & 27 & 1 & 2 \\
\hline 4 & 4 & 6 & 4 & 10 & 10 & 5 & 16 & 16 & 16 & 6 & 16 & 22 & 22 & 3 & 8 & 28 & 28 & 2 & 1 \\
\hline 5 & 5 & 5 & 8 & 11 & 11 & 3 & 4 & 17 & 17 & 4 & 2 & 23 & 23 & 1 & 4 & 29 & 29 & 1 & 1 \\
\hline 6 & 6 & 4 & 8 & 12 & 12 & 4 & 16 & 18 & 18 & 5 & 4 & 24 & 24 & 4 & 1 & 30 & 30 & 1 & 16 \\
\hline
\end{tabular}

\section{Evaluation and Comparison}

To evaluate the rate efficiency of the proposed codes, it is necessary to analyze data shown in Table 1. The first thing we notice from this table is an excellent agreement between the theory and experiments. More precisely, we see that for byte lengths $b=3,4,5,7,9$ and 11 bits, we can construct six codes reaching the bound given in Theorem 2. These codes are either perfect $((30,25),(126,119)$ and $(2046,2035))$, or optimal $((6,3),(12,8)$ and $(504,495))$ in the sense of maximum rate. On the other hand, we also see that for byte lengths $b=6,8,10$ and 12 bits we cannot construct perfect or optimal codes.

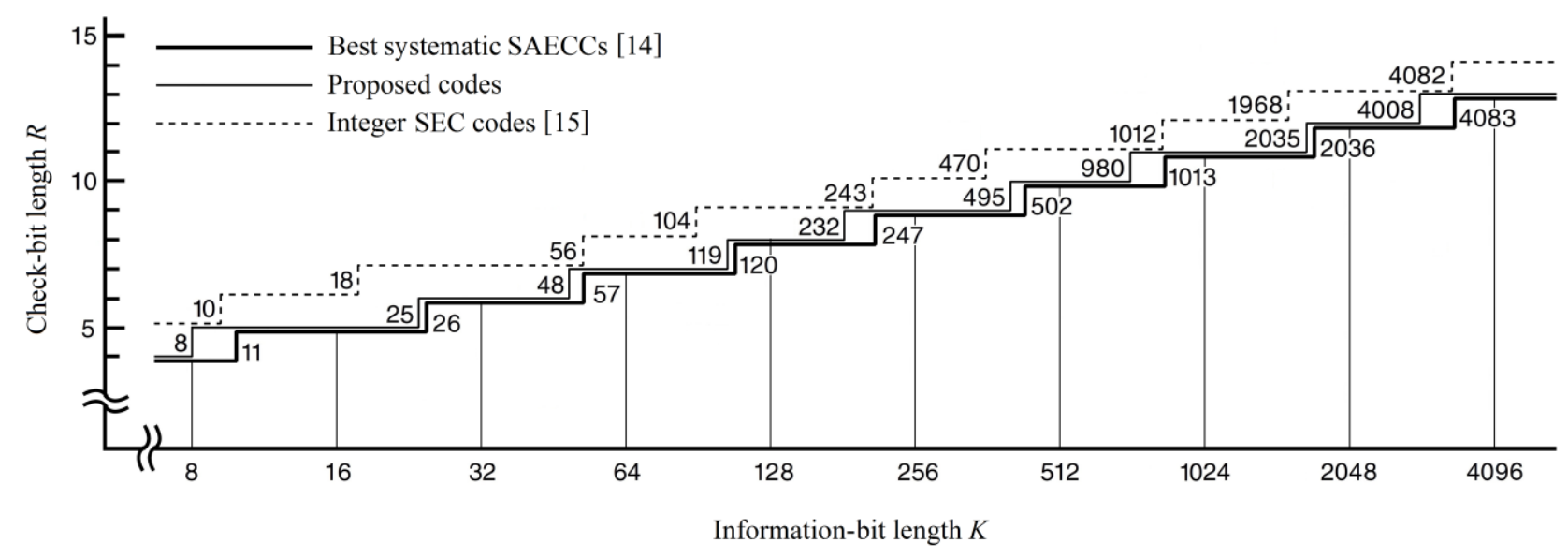

Fig. 2. Comparison of information-bit lengths and check-bit lengths of the best systematic SAECCs [14], the proposed codes and integer SEC codes [15].

Despite this shortcoming, the presented codes are very efficient in terms of redundancy. To illustrate this, in Fig. 2, they are compared with codes from [14] and [15]. As we can see, for practical data lengths up to 4096 bits, the proposed codes require the same number of check-bits as the best systematic SAECCs [14] and one check-bit less than integer single error correcting (SEC) codes [15]. Besides this, from Fig. 2, it can be observed that perfect integer SAECCs have slightly lower rates than the codes from [14]. The reason for this is that the proposed codes are defined over an alphabet $\left\{0,1, \ldots, 2^{b}-2\right\}$, which is a subset of the set $\left\{0,1, \ldots, 2^{b}-1\right\}$.

On the other hand, the main advantage of the proposed codes over the best systematic SAECCs lies in the ability to faster encode/decode data bits. Namely, from [16] we know that any integer encoder/decoder must perform approximately $b \cdot K$ operations per $K$-bit data word. In 
contrast to this, from (1) we observe that the MGT encoder/decoder executes two operations at the bit level: one multiplication between $d_{i}$ and $u_{i}\left(\left\lceil\log _{2}(K+1)\right\rceil\right.$ operations $)$ and one addition between two $\left\lceil\log _{2}(K+1)\right\rceil$ - bit integers $\left(\left\lceil\log _{2}(K+1)\right\rceil\right.$ operations). Considering that there exist $K$ data bits, we easily come to the conclusion that the MGT encoder/decoder must perform approximately $K \cdot \log _{2} K$ operations per $K$-bit data word. This means that the encoding/decoding complexity grows linearithmic with the data length, while in the case of proposed codes it increases linearly.

\section{Conclusion}

This paper proposed a class of integer codes capable of correcting single asymmetric errors. The proposed codes are constructed with the help of a computer and are very close to being optimal in terms of redundancy. The results of an exhaustive search have shown that, for practical data lengths up to 4096 bits, the proposed codes use the same number of check-bits as the best systematic single asymmetric error correcting codes. In addition, it has been shown that, for some lengths the proposed codes are perfect. The parameters of these codes are $\left(2^{b}-2,2^{b}-b\right.$ -2), which makes them one of the most rate-efficient codes in the literature.

\section{References}

[1] J. M. Borden, "Optimal Asymmetric Error Detecting Codes," Inf. Control, vol. 53, nos. 1-2, pp. 66-73, Apr.-May. 1982.

[2] S. D. Constantin and T. R. N. Rao, "On the Theory of Binary Asymmetric Error Correcting Codes," Inf. Control, vol. 40, no. 1, pp. 20-26, Jan. 1979.

[3] R. W. Hamming, "Error Detecting and Error Correcting Codes," Bell Syst. Tech. J., vol. 29, no. 2, pp. 147-150, Apr. 1950.

[4] R. R. Varshamov and G. M. Tenengol'ts, "Correction Code for Single Asymmetric Errors," Automat. Telemekh., vol. 26, no. 2, pp. 288-292, Feb. 1965.

[5] P. Delsarte and P. Piret, "Bounds and Constructions for Binary Asymmetric ErrorCorrecting Codes," IEEE Trans. Inform. Theory, vol. 27, no. 1, pp. 125-128, Jan 1981.

[6] A. Shiozaki, "Single Asymmetric Error-Correcting Cyclic AN Codes," IEEE Trans. Comput., vol. 31, no. 6, pp. 554-555, June 1982.

[7] J. Weber, C. DeVroedt and D. Boekee, "Bounds and Construction for Binary Codes of Length Less than 24 and Asymmetric Distance Less than 6," IEEE Trans. Inform. Theory, vol. 34, no. 5, pp. 1321-1331, Sept. 1988.

[8] Z. Zhang and X. Xia, "New Lower Bounds for Binary Codes of Asymmetric Distance Two," IEEE Trans. Inform. Theory, vol. 38, no. 5, pp. 1592-1597, Sept. 1992.

[9] S. Al-Bassam, R. Venkatesan and S. Al-Muhammadi, "New Single Asymmetric Error Correcting Codes," IEEE Trans. Inform. Theory, vol. 43, no. 5, pp. 1619-1623, Sept. 1997.

[10] S. Al-Bassam and S. Al-Muhammadi, "A Single Asymmetric Error-Correcting Code with $2^{13}$ Codewords of Dimension 17," IEEE Trans. Inform. Theory, vol. 46, no. 1, pp. 269-271, Jan. 2000.

[11] F. Fu, S. Ling and C. Xing, "New Lower Bounds and Constructions for Binary Codes Correcting Asymmetric Errors," IEEE Trans. Inform. Theory, vol. 49, no. 12, pp. 32943299, Dec. 2003. 
[12] M. Grassl, P. Shor, G. Smith, J. Smolin and B. Zeng, "New Constructions of Codes for Asymmetric Channels Via Concatenation", IEEE Trans. Inform. Theory, vol. 61, no. 4, pp. 1879-1886, Apr. 2015.

[13] B. Bose and S. Al-Bassam, "On Systematic Single Asymmetric Error Correcting Codes," IEEE Trans. Inform. Theory, vol. 46, no. 2, pp. 669-672, Mar. 2000.

[14] K. Abdel-Ghaffar and H. Ferreira, "Systematic Encoding of the Varshamov-Tenengol'ts Codes and the Constantin-Rao Codes," IEEE Trans. Inform. Theory, vol. 43, no. 1, pp. 340354, Jan. 1997.

[15] A. Radonjic, “(Perfect) Integer Codes Correcting Single Errors," IEEE Commun. Lett., vol. 22, no. 1, pp. 17-20, Jan. 2018.

[16] A. Radonjic and V. Vujicic, "Integer Codes Correcting Burst Errors within a Byte," IEEE Trans. Comput., vol. 62, no. 2, pp. 411-415, Feb. 2013. 